\title{
Implementation of Fuzzy Multiple Objective Decision Making Algorithm in a Heterogeneous Mobile Environment
}

\author{
P.M.L. Chan ${ }^{1}$, Y.F. $\mathrm{Hu}^{2}$, R.E. Sheriff ${ }^{1}$, \\ Department of Electronics and Telecommunications ${ }^{1}$ \\ Department of Cybernetics, Internet and Virtual Systems ${ }^{2}$ \\ University of Bradford, UK
}

\begin{abstract}
Mobile satellite networks are now being utilized to provide complementary service coverage to their terrestrial counterparts. The migration from voice-dominated service delivery to a data-centric environment implies that the choice of one particular access network over another will need to take into account many different criteria, which previously need not have been considered. For this reason, it is important that a suitable decision making algorithm is designed to ensure that all of the different characteristics of the system are taken into account when making the segment selection for a particular service delivery. In this paper, a segment selection algorithm based on the fuzzy multiple objective decision making algorithm is presented. This algorithm is analyzed to show how the overall performance of a multi-access segment network can be improved by varying the relative importance of certain service and network characteristics.
\end{abstract}

\section{INTRODUCTION}

Mobile satellite networks are now being utilized to provide complementary service coverage to their terrestrial counterparts. At present, the services on offer to the mobile user are limited to voice and low data rate applications, be it via terrestrial or satellite modes of delivery. Such a service scenario is set to change with the introduction of evolved second-generation networks, such as the GSM derivative GPRS, followed closely by third-generation (3G) mobile technologies. Shortly, the sophisticated mobile user will be able to access a multitude of new services and applications at data rates of $144 \mathrm{kbit} / \mathrm{s}$ and beyond, incorporating packetoriented as well as traditional circuit-switched forms of delivery. It is anticipated that future-generation mobile technologies will rely on both satellite and terrestrial networks to deliver services with the desired Quality of Service (QoS).

In comparison to today's mobile networks, in the future, the mobile user should have a much greater choice not only in terms of the type of services and applications on offer but also the preferred mode of delivery, for example, either through terrestrial or satellite access networks. It can be envisaged that the user may wish to trade-off cost against quality, for example, or perhaps to determine the proposed access network according to the application on offer. Similarly, network operators may be able to arrange a choice of service packages that are catered towards particular categories of user, for example, a business user would have a different service profile in comparison to a tourist.
Currently, in areas where a satellite is operating alongside a terrestrial network, the terrestrial network will always be given priority when establishing a call. In the near future, however, the selection of a certain access network for the initiation of a particular communication session could rely on a sophisticated algorithm, incorporating not only the basic QoS criteria, such as signal quality, but also other considerations, such as the mobile terminal's available battery power, required bandwidth, service priority and so on. Here, it can be seen that the choice of one network over another is no longer black or white but rather depends on a number of inter-related parameters. Ideally, it should be possible to maximize the efficiency of the heterogeneous environment by taking into account the various considerations that need to made when establishing a session, such that both terrestrial and satellite resources are optimally utilized.

In this paper, the concepts of fuzzy logic are applied to a future heterogeneous mobile environment, in which terrestrial and satellite mobile networks operate alongside each other. The use of fuzzy logic allows a sensitivity analysis to be performed in order to determine which factors are critical to the efficient utilization of the network. In this paper, two specific parameters are given particular attention: Charging Model and Quality of Service.

The paper is organized as follows: In the next section, a brief introduction to multiple objective decision making (MODM) is presented followed by a discussion of relevant segment selection criteria. The constituents of the algorithm are described in Section III, followed by a description of the proposed algorithm in Section IV. The simulation and analysis of the proposed algorithm is then presented before drawing conclusion on the algorithm's performance.

\section{MULTIPLE OBJECTIVE DECISION MAKING}

In multiple objective decision problems, all alternatives available to the system are evaluated according to a number of criteria. Each criterion will induce a particular ordering of the alternatives and a relevant procedure is then implemented to construct one overall preference ordering [1]. There are many variations on the methods used for MODM. The algorithm proposed here uses the model proposed by Yager in [2]. This method is chosen since it is suitable for solving 
problems where the objectives are of varying degree of importance. Detailed descriptions of other types of MODM models can be found in [3] and [4]. In general, MODM can be implemented in any system where various alternatives and criteria exist. However, this methodology has yet to be implemented in a heterogeneous wireless environment. Currently, the primary application of MODM lies in control and industrial engineering.

\section{CONSTITUENTS OF ALGORITHM}

The segment selection criteria is important as it influences the choice of the target segment. As mentioned previously, several criteria, which can include user-based QoS parameters and other network characteristics, can be considered in the proposed algorithm. For example, in addition to the signal strength, other parameters such as the available bandwidth, reliability, latency and network coverage can be taken into account. When considering userbased parameters, the types of application, charging model and the preferred and prohibited user segment can be incorporated in the algorithm. If the change of segment occurs when the user is currently using another segment, the QoS perceived by the user can also be considered in the algorithm.

Seven example criteria are used in the algorithm described in this paper, namely, signal strength, bandwidth, cost, reliability, latency, battery status and the user's preferred segment (priority). This can be seen in Fig. 1, where the input from the system is considered. In addition to the criteria mentioned above, several non-fuzzy or crisp criteria could also be considered in the algorithm such as the availability of segment and also the terminal type. These criteria are considered as crisp sets since a segment can only be classed as either being available or not, and not both simultaneously. A similar argument applies to terminal type.

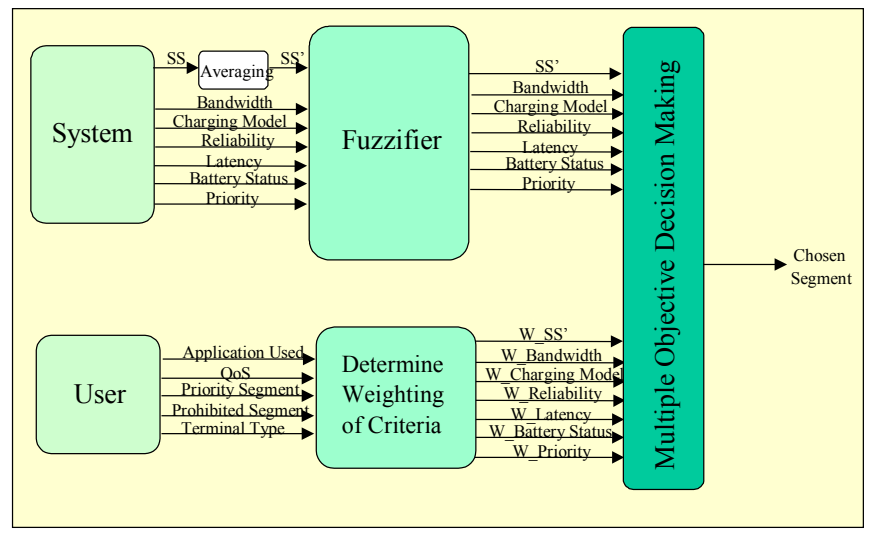

Fig. 1. Block Diagram for Segment Selection

The role of the user in the algorithm is detailed in the next section. Generally, the input from the user is used to determine the weighting given to each of the seven criteria. This input, for instance, could be the importance of cost compared to the importance of received quality of service.
Other parameters could include the type of applications used and the choice of priority or prohibited segments. As an example, the user can be provided with the following set of questions, as illustrated in Fig. 2.

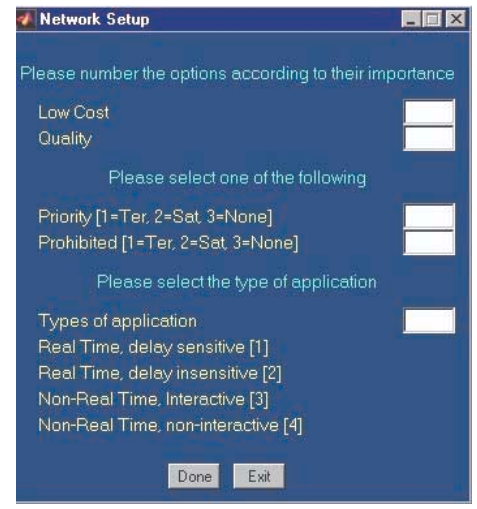

Fig. 2. An Example User Interface

The main purpose of the segment selection algorithm is to select a segment for a particular service which can satisfy the following objectives; low cost, good signal strength, optimum bandwidth, low network latency, high link availability, long battery life, while taking into account the preferred segment of the user. There are two different stages in the algorithm.

The fuzzification procedures and the weighting of the criteria are performed in the first stage. The former involves the evaluation and comparison of the available segments, whereas the latter is used to evaluate the importance of each criteria based on the instructions received from the network provider and the user.

\section{SEGMENT SELECTION ALGORITHM}

In the first stage, data from the system are fed into a fuzzifier, to be converted into fuzzy sets. A fuzzy set is a set without a crisp, clearly defined boundary and therefore, has a varying degree of membership. This is different from a crisp set in which elements are only considered members of a set if its membership is assigned a value of 1 . For example, if the charging model is considered in a crisp set, the cost of using a segment can only be classed as expensive or inexpensive. If it is classed as expensive, it will be given a membership value of "1"; otherwise, it will have a membership value of "0". However, in a fuzzy set, the cost of a segment can be represented by anything between " 0 " and " 1 " depending on the membership function. The membership function is a curve or line that defines how each datum or value is mapped onto a membership value. In order to obtain the membership values for the fuzzy sets, the measurements for a particular parameter are mapped onto a membership function.

As an example, if the objective of the target segment is low cost, a membership function with the objective "low cost" can be constructed, as shown in Fig. 3a. From the diagram, it can be inferred that if cost is low, the membership values will 
be high. Note that the $\mathrm{x}$-values for cost are normalized between " 0 " and " 1 ".

In the same diagram, the method by which the membership values are assigned is demonstrated. If the normalized cost for UMTS is 0.5 , the membership value for UMTS is 0.8 . The same principle applies to all three segments.

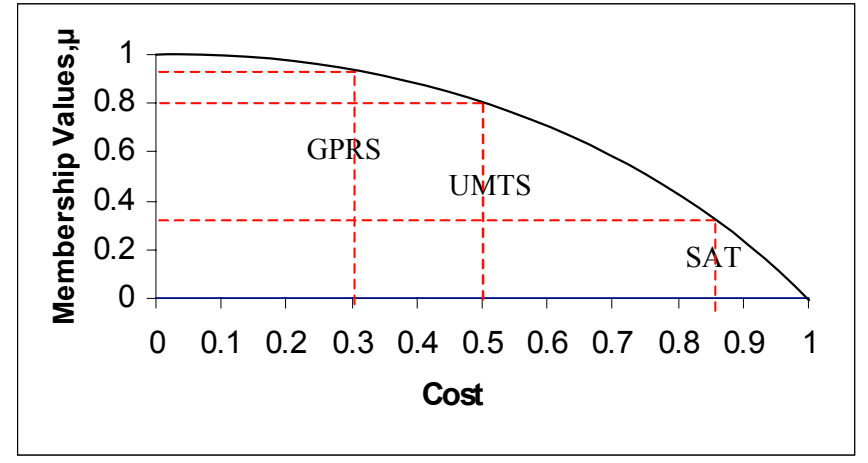

(a) Objective: Low Cost

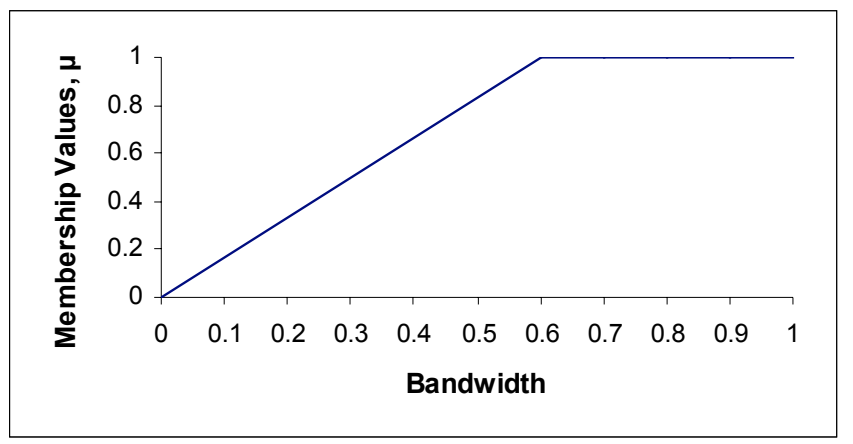

(a) Objective: High Bandwidth

Fig. 3. Membership Function Cost and Bandwidth

On the other hand, the weighting of criteria is developed by obtaining a ratio scale for the criteria based upon a paired comparison of each criterion. This ratio scale is influenced by the user's preference in terms of cost, quality and application used. This procedure is also known as the analytic hierarchy process (AHP), which was developed by Saaty [5] for allocating resources according to priority levels. AHP consists of three main principles: decomposition of the decision problem, comparative judgment of the elements and synthesis of the priorities. In the first step, the problem is decomposed into its constituent parts (identification of segment selection criteria). The second step is the comparison of the criteria. Here, the criteria are compared in pairs. Finally, in the third step, the weightings for all the criteria are obtained using the eigenvector method. The procedures involved in obtaining the weighting are described below:

Assume that $C=\left\{C_{1}, \ldots, C_{p}\right\}$ is a set of $p$ decision criteria. A set of values of relative importance is then assigned to each criterion by performing a pairwise comparison between the designated criterion and the other criteria. For example, if $C_{I}$ is the designated criterion, then the set of values of relative importance for $C_{l}$ in comparison with the other criteria can be written as $\left\{a_{12}, a_{13}, \ldots a_{1 p}\right\}$, where $a_{1 j, j \neq 1}$ is determined by comparing the importance of criteria $C_{l}$ to $C_{j}$. The values of $a_{l j}$ are determined by the system designer based on the network capability, service requirements and the user's input. The definition of the values assigned for the comparison is shown in Table 1.

TABLE 1

DEFINITION FOR COMPARING IMPORTANCE OF CRITERIA [5]

\begin{tabular}{|c|c|}
\hline Importance & Definition \\
\hline 1 & Equal Importance \\
\hline 3 & Weak importance of one over the other \\
\hline 5 & Strong importance of one over another \\
\hline 7 & Demonstrated importance of one over the other \\
\hline 9 & Absolute importance of one over the other \\
\hline $2,4,6,8$ & Intermediate values; two adjacent judgements \\
\hline
\end{tabular}

Then a matrix, $\boldsymbol{B}$, of dimension $p \mathrm{x} p$ can be created where:

$$
\begin{aligned}
& b_{i i}=1 \\
& b i j=a i j, i \neq j \\
& b j i=1 / b i j,
\end{aligned}
$$

An example of the comparison is shown in the following matrix. Here, criterion $C_{1}$ is first compared to $C_{2}, C_{3}$ and so on. In [2] , it was shown that by finding the unit eigenvector, $\boldsymbol{W}$, corresponding to the maximum eigenvalue of $\boldsymbol{B}$ produces the cardinal ratio scale of the compared elements. In this example, $\mathrm{C}_{2}$ has a higher importance level when compared to $\mathrm{C}_{1}$. However, it is given the same importance in comparison to the other five criteria.

$$
\begin{aligned}
& \begin{array}{lllllll}
C_{1} & C_{2} & C_{3} & C_{4} & C_{5} & C_{6} & C_{7}
\end{array}
\end{aligned}
$$

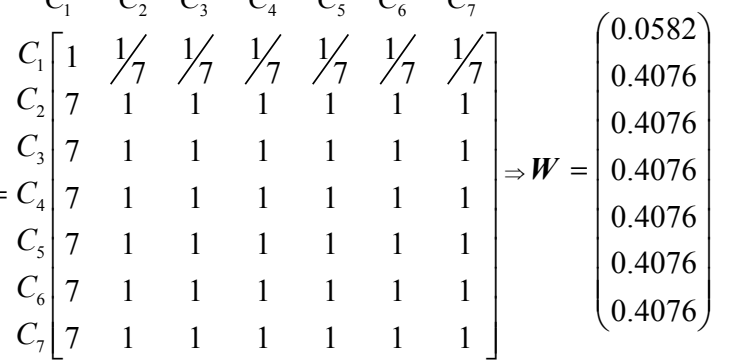

To obtain the final weighting matrix, $\alpha$, used in the decision process, $\boldsymbol{W}$ is multiplied with the number of criteria, $n$.

$$
\alpha=n \boldsymbol{W}=\left(\begin{array}{l}
0.4076 \\
2.8529 \\
2.8529 \\
2.8529 \\
2.8529 \\
2.8529 \\
2.8529
\end{array}\right)
$$

The second stage of the algorithm uses the methodology introduced in [2]. In this stage, the weightings are applied to each criterion. This is performed by raising the corresponding 
elements in the fuzzy set to the corresponding matrix elements in $\alpha$. A decision function is then obtained, which can simultaneously satisfy all the decision objectives. This is given by the intersection of all objectives sets, such that:

$$
D=C_{1}{ }^{\alpha_{1}} \cap{C_{2}}^{\alpha_{2}} \cap{C_{3}}^{\alpha_{3}} \cap{C_{4}}^{\alpha_{4}} \ldots \cap C_{n}{ }^{\alpha_{n}}
$$

Several rules are important when considering fuzzy logic. The AND (intersection) of any two fuzzy variables results in the minimum truth value, whereas the OR (union) of two fuzzy variables results in the maximum truth value.

$$
\begin{aligned}
& \mu_{A \cap B}=\min \left(\mu_{A}, \mu_{B}\right) \\
& \mu_{A \cup B}=\max \left(\mu_{A}, \mu_{B}\right)
\end{aligned}
$$

One decision function can be calculated for each segment and since $D$ is obtained using the minimum truth value, the chosen segment is the segment with the maximum or highest membership values of $D$.

\section{Performance EVALUATION}

\section{A. Testbed}

Three wireless access segments are considered in the the experiments that follow; General Packet Radio Service (GPRS), Universal Mobile Telecommunications System (UMTS) and a geostationary satellite system. GPRS is a service designed to transfer non real-time services over existing GSM radio networks, whereas UMTS is capable of supporting both non real-time and real-time services with data rates of up to $2 \mathrm{Mbps}$. The satellite system considered here is capable of supporting user data rates in the range corresponding to those specified for Satellite-UMTS, that is up to $144 \mathrm{kbps}$ in a mobile environment.

\section{B. Experiment 1}

Two sets of experiments are performed. In the first experiment, the same weightings are applied to all seven criteria. The main focus of this experiment is to define a set of conditions that will make the satellite segment to be competitive with its terrestrial counterparts, despite the fact that it is significantly more expensive, as indicated by the value of the charging model value $\left(\mathrm{C}_{1}\right)$. This will be achieved by offering services at a greater bandwidth. The investigation aims to determine the minimum bandwidth $\left(\mathrm{C}_{2}\right)$ required by the satellite segment that is required to make it a possible target segment. With this information, the network operator can then have an indication of how certain criteria or network characteristics for a particular segment can be optimized to make it on par with the other wireless segments. The service provider can subsequently use this information when specifying the characteristics of each segment.

The parameters for Experiment 1 are shown in Table 2. Here, the membership function for $\mathrm{C}_{2}$ is not included, since this is to be determined by the experiment. As noted previously, the values for $\alpha$ are obtained by providing equal importance to all the seven criteria. The criteria are rated from a scale of 0 to 1 , with " 0 " being the least likely to satisfy an objective and " 1 " being the most likely to meet the requirement of an objective. To be more precise, if the charging model is considered, " 0 " indicates a very expensive segment whereas a rating of " 1 " shows that the segment is inexpensive. These parameters can be obtained using the membership functions shown in Fig. 3. Also note that other than cost, the other criteria have been assigned membership values of 0.9 , so as not to influence the comparison between bandwidth and cost.

TABLE 2

PARAMETERS FOR EXPERIMENT 1

\begin{tabular}{|l|c|c|c|c|c|}
\hline \multicolumn{2}{|c|}{ Criteria } & \multirow{2}{*}{$\alpha$} & \multicolumn{3}{c|}{ Membership Values } \\
\cline { 4 - 6 } & & GPRS & UMTS & SAT \\
\hline $\begin{array}{l}\text { Charging } \\
\text { Model }\end{array}$ & $\mathrm{C}_{1}$ & 2.6458 & 0.9 & 0.6 & 0.3 \\
\hline Latency & $\mathrm{C}_{3}$ & 2.6458 & 0.9 & 0.9 & 0.9 \\
\hline Reliability & $\mathrm{C}_{4}$ & 2.6458 & 0.9 & 0.9 & 0.9 \\
\hline $\begin{array}{l}\text { Signal } \\
\text { Strength }\end{array}$ & $\mathrm{C}_{5}$ & 2.6458 & 0.9 & 0.9 & 0.9 \\
\hline Battery & $\mathrm{C}_{6}$ & 2.6458 & 0.9 & 0.9 & 0.9 \\
\hline $\begin{array}{l}\text { Segment } \\
\text { Priority }\end{array}$ & $\mathrm{C}_{7}$ & 2.6458 & 0.9 & 0.9 & 0.9 \\
\hline
\end{tabular}

Since the intersection function is used to determine the target segment, the minimum membership value of the chosen criteria has the most influence on the target segment. Therefore, for the satellite segment to be chosen, the following condition must hold true. This is obtained by applying equation (5) to (4).

$$
\begin{aligned}
& \operatorname{Min}\left[C_{1}^{\alpha}(S A T), C_{2}^{\alpha}(S A T), C_{3}^{\alpha}(S A T), \ldots C_{n}^{\alpha}(S A T)\right]> \\
& \operatorname{Min}\left[C_{1}^{\alpha}(G P R S), C_{2}^{\alpha}(G P R S), C_{3}^{\alpha}(G P R S), \ldots C_{n}^{\alpha}(G P R S)\right] A N D \\
& \operatorname{Min}\left[C_{1}^{\alpha}(S A T), C_{2}^{\alpha}(S A T), C_{3}^{\alpha}(S A T), \ldots C_{n}^{\alpha}(S A T)\right]> \\
& \operatorname{Min}\left[C_{1}^{\alpha}(U M T S), C_{2}^{\alpha}(U M T S), C_{3}^{\alpha}(U M T S), \ldots C_{n}^{\alpha}(U M T S)\right]
\end{aligned}
$$

In experiment 1 , the membership value of $C_{1}$ for the satellite segment is given as 0.3 . Therefore, for the satellite segment to be selected,

$\mathrm{C}_{2}{ }^{\alpha}(\mathrm{SAT})>0.3^{2.6458}$ AND $\mathrm{C}_{2}{ }^{\alpha}(\mathrm{GPRS}) \leq 0.3^{2.6458}$ AND
$\mathrm{C}_{2}{ }^{\alpha}(\mathrm{UMTS}) \leq 0.3^{2.6458}$

The same argument can be applied for the UMTS and GPRS segments.

\section{Experiment 2}

In the Experiment 2, different weightings are then applied to the criteria to demonstrate the influence of the weightings on the final decision. In particular, the weighting of bandwidth is given a demonstrated importance over cost. Therefore, it can be assumed that the application is tailored to suit a business user.

Table 3 shows the weighting applied to each criterion. Two scenarios are considered in this simulation: 1) both cost and bandwidth are of equal weighting; 2) Bandwidth has a higher 
weighting than cost. The matrix used to obtain $\alpha$ for Scenario 2 is the same as the matrix example shown in Section IV. The membership values used in this experiment are the same as those assigned to Experiment 1.

TABLE 3

PARAMETERS FOR EXPERIMENT 2

\begin{tabular}{|l|l|c|c|}
\hline \multicolumn{2}{|c|}{ Criteria } & \multicolumn{2}{c|}{ Weightings, $\alpha$} \\
\cline { 3 - 4 } \multicolumn{2}{|l|}{} & Scenario 1 & Scenario 2 \\
\hline $\begin{array}{l}\text { Charging } \\
\text { Model }\end{array}$ & $\mathrm{C}_{1}$ & 2.6458 & 0.4076 \\
\hline Bandwidth & $\mathrm{C}_{2}$ & 2.6458 & 2.8529 \\
\hline Latency & $\mathrm{C}_{3}$ & 2.6458 & 2.8529 \\
\hline Reliability & $\mathrm{C}_{4}$ & 2.6458 & 2.8529 \\
\hline $\begin{array}{l}\text { Signal } \\
\text { Strength }\end{array}$ & $\mathrm{C}_{5}$ & 2.6458 & 2.8529 \\
\hline Battery & $\mathrm{C}_{6}$ & 2.6458 & 2.8529 \\
\hline $\begin{array}{l}\text { Segment } \\
\text { Priority }\end{array}$ & $\mathrm{C}_{7}$ & 2.6458 & 2.8529 \\
\hline
\end{tabular}

The results obtained for Experiment 2 are shown in Table 4. Again, the results shown are aimed at selecting the satellite segment as the most suitable segment since this segment is considered the most undesirable when the charging model is first taken into consideration.

TABLE 4

RESULT FOR EXPERIMENT 2

\begin{tabular}{|c|c|c|c|c|c|c|c|c|}
\cline { 2 - 9 } \multicolumn{1}{c|}{} & \multicolumn{4}{c|}{ Equal Weighting } & \multicolumn{3}{c|}{ Higher Weighting for $\mathrm{C}_{2}$} \\
\cline { 2 - 9 } & $\begin{array}{c}\text { Charging } \\
\text { Model }\end{array}$ & \multicolumn{2}{c|}{ Bandwidth } & \multicolumn{2}{c|}{$\begin{array}{c}\text { Charging } \\
\text { Model }\end{array}$} & \multicolumn{2}{c|}{ Bandwidth } \\
\cline { 2 - 9 } & $\mu\left(\mathrm{C}_{1}\right)$ & $\mathrm{C}_{1}{ }^{\alpha}$ & $\mathrm{C}_{2}{ }^{\alpha}$ & $\mu\left(\mathrm{C}_{2}\right)$ & $\mu\left(\mathrm{C}_{1}\right)$ & $\mathrm{C}_{1}{ }^{\alpha}$ & $\mathrm{C}_{2}{ }^{\alpha}$ & $\mu\left(\mathrm{C}_{2}\right)$ \\
\hline GPRS & 0.900 & 0.757 & 0.041 & 0.299 & 0.900 & 0.958 & 0.612 & 0.842 \\
\hline UMTS & 0.600 & 0.258 & 0.041 & 0.299 & 0.600 & 0.812 & 0.612 & 0.842 \\
\hline SAT & 0.300 & 0.041 & 0.042 & 0.302 & 0.300 & 0.612 & 0.613 & 0.843 \\
\hline
\end{tabular}

The membership value of $C_{1}\left(\mu\left(C_{1}\right)\right)$ in Table 4 is obtained from the simulation parameters shown in Table $2 . \mathrm{C}_{1}{ }^{\alpha}$ is then calculated by using the values of $\alpha$ in Table 3 . In order to make the satellite segment the preferred segment, $\mathrm{C}_{2}{ }^{\alpha}$ values for GPRS and UMTS have to be set to be equal or less than the minimum value of $\mathrm{C}_{1}{ }^{\alpha}$ for the satellite segment, as explained in Experiment 1.

Therefore, in the first scenario, where equal priority and hence weighting is implemented, the maximum value of $\mathrm{C}_{2}{ }^{\alpha}$ permissible in both GPRS and UMTS in order for the satellite segment to be selected is 0.041 , which coincides with the minimum value of $\mathrm{C}_{1}{ }^{\alpha}$ in the satellite segment. The required membership values of $\mathrm{C}_{2}$ for GPRS and UMTS are then found to be 0.299 , whereas for the satellite segment, this value is 0.302 .

On the other hand, in the second scenario, when a higher priority (weighting) is assigned to bandwidth, the required membership values of $\mathrm{C}_{2}$ for GPRS and UMTS are both
0.842. For the satellite segment, this is 0.843 . In contrast to the first scenario, in order for the satellite segment to be selected, the available bandwidth to be provided need not be significantly more than that provided by the UMTS or GPRS segments. In other words, when equal priority is assigned to all criteria, the selection of the satellite segment is in practice, unlikely. However, from Experiment 2, it can be seen that when the user expresses a preference of bandwidth against cost, the satellite segment can provide a viable alternative to its terrestrial counterparts.

\section{CONCLUSION}

This paper has presented a segment selection algorithm based on the concept of fuzzy multiple objective decision making (MODM). This method is suitable for the segment selection algorithm as it provides approaches to making decisions in complex situations where more than one decision objective should be considered. The results show that even though the access segments utilized in the algorithm might have different behaviors, it is possible to choose a segment that was initially thought to be unsuitable by adjusting the characteristics of other criteria. Furthermore, the significance of the weighting method and the conditions required to make a segment more attractive to the user has also been discussed.

The next stage of the work involves improving the method in which each of the criteria is ranked based on the actual performance measurements of each network. In addition, further analysis involving a larger number of criteria to be compared could be investigated.

\section{ACKNOWLEDGEMENT}

This article is based on work that was performed as part of the SUITED project, which is partially funded by the European Commission under the Information Society Technologies (IST) Program.

\section{REFERENCES}

[1] G.J. Klir, B. Yuan, Fuzzy Sets and Fuzzy Logic, Prentice Hall P T R, USA, 1995.

[2] R.R. Yager, "Multiple Objective Decision Making using Fuzzy Sets", International Journal of Man-Machine Studies, Vol. 9, pp. 375-382, 1977.

[3] R. Fuller, C. Carlsson, "Fuzzy Multiple Criteria Decision Making: Recent Developments", Fuzzy Sets and Systems, Vol. 78, pp. 139-153, 1996.

[4] T.J. Ross, Fuzzy Logic with Engineering Applications, McGraw-Hill, Inc., USA, 1995.

[5] T.L. Saaty, The Analytic Hierarchy Process: Planning, Priority Setting, Resource Allocation, McGraw-Hill, Inc., USA, 1980. 